\title{
A Study on Open-Source Wiki Platforms
}

\author{
Lixăndroiu Radu \\ lixi.radu@,unitbv.ro \\ Maican Cătălin \\ maican@unitbv.ro \\ Dovleac Lavinia \\ lavina.dovleac@unitbv.ro \\ Transilvania University of Brasov, Romania
}

\begin{abstract}
In this study, the authors are looking for the latest trends in choosing an open-source wiki platform for document management. To rigorously substantiate the choice of the most appropriate platform for each case, the authors propose a mathematical model of comparison, based on the similarity of the platform characteristics with the ideal platform. Mainly, it is considered that in each situation we can identify the determined characteristics of the most appropriate platform (the ideal platform), characteristics that are then compared with the characteristics of the existing platforms on the market. The most suitable platform is the one with the highest score, meaning it is the more likely to the searched platform.
\end{abstract}

Keywords: wiki platform, Jaccard index, similarity indices

Jel Code: M15

\section{Introduction}

Wiki applications are web applications that allow users to add content and keep their own successive versions, similar to an Internet forum, but it also allows anyone else to modify the content. Wiki applications are part of the phenomenon called Web 2.0. The term Wiki also denotes the collaborative software used to create such a website.

Techniques and methods of Wiki applications are used by some domestic companies to collect and provide information and documentation. According to Wiki principles, information can be placed on the Wiki server by employees involved in the company's various processes. This kind of internal projects are called Enterprise Wiki or Corporate Wiki. Being internal information, they are usually made available to employees only, within an intranet of the company. If necessary, a limited access can also be given to non-corporate users within a protected extranet.

The required Wiki software can be obtained from many sources, both free (Open Source) and commercial.

Several advantages of a Wiki inside an enterprise are: the ability to create a user-friendly, unitary and very current multimedia documentary of all enterprise processes / products / projects, including archiving and version control; reducing email traffic; increasing the efficiency of internal communication; increasing employee identification with the enterprise. Some disadvantages are: the initial cost of implementing a Wiki system; the time needed by the employees to keep up with all Wiki content (but less time for traditional documentation).

Business Wikis are used by many of the world's innovative companies for team collaboration, document co-author, management and intranet, planning and contacting people, and for project work.

Fields of wiki usage

- for document co-author

- for textbooks

- as a database

- for intranet and / or extranet development 
- for communication initiatives

- to display static and dynamic information

- to display answers to recent questions (FAQs)

- for an online topic chat feature

- to add requirements

- To store college notes

- Collect sources of information in a single accessible place from anywhere

- for team communication

- to access the software issues source

- to organize / manage projects

- to organize events

- To publish articles

- to publish checklists

- To publish the reference documents

- To record appointment notes

- To register a team or organization

- to resolve problems remotely

- to track deadlines

- to track invoices

- for brainstorming and mindmapping

- to create to-do lists

- for a journalistic conversation

- for record keeping

- for journals

- Create research notes

- plan and write novels

\section{The Need for a Wiki Platform / Platform Requirements}

The first step in finding the most appropriate wiki platform regarding a number of identified needs was to find the most representative wiki platforms on the market. The authors have conducted an exploratory research, looking for platforms that meet the identified requirements. Although the requirements to be met by the platform were previously identified, some platforms that only partially met these requirements were selected for comparison. The motivation was represented by the use of the Discosure index computation (DIC) model - the simple cardinal valuation method, which gave different values according to the importance of the criteria.

The top 24 wiki platforms identified are:

1. BlueSpice MediaWiki

2. Central Desktop

3. ConnectedText

4. DokuWiki

5. FlexWiki

6. Gitit

7. IBM Connections

8. Midgard Wiki

9. MindTouch

10. PBworks

11. PhpWiki

12. PmWiki
13. SharePoint

14. Socialtext

15. Swiki

16. TiddlyWiki

17. Tiki Wiki CMS Groupware

18. Trac

19. TWiki

20. UseModWiki

21. Wagn

22. Wiki.js

23. XWiki

24. ZWiki 
In the given situation, a free wiki platform with GPL software license, open source, multilingual, programming language PHP, ASP.NET or C \#, intranet, scientific, technical, mathematical, file uploading, attachments etc.). The requirements of the research team related to the Wiki platform (closely related to the individual problem) were the following:

1. GPL Software license

2. Open source

3. Multilingual

4. Programming language PHP, ASP.NET or C\#

5. Public

6. Private

7. Corporate

8. Enterprise

9. Education

10. Intranet

11. Personal

12. Scientific, technical, mathematical

13. File uploading

14. Attachments

15. Spam prevention

16. Page access control

17. Inline HTML

18. User-customizable interface

19. Document renaming

20. Web feeds

21. Export, import

22. Extensibility

23. Selectable wiki syntax

24. Wiki farms

\section{Similarity indices - Jaccard index}

Jaccard index, also known as the Jaccard similarity coefficient is a statistical indicator used to compare the similarity of several sets. Jaccard index of similarity measure between sets of finite sentences, and is defined as the intersection of the sets divided by the sample sets meeting:

$I(A, B)=\frac{|A \cap B|}{|A \cup B|}$

If $\mathrm{A}$ and $\mathrm{B}$ are empty, define $J(A, B)=1$

$0 \leq J(A, B) \leq 1$

The Jaccard distance measures the dissimilarity between sample sets. The Jaccard distance is complementary to the Jaccard coefficient and is obtained by subtracting the Jaccard coefficient from 1:

$d_{J}(A, B)=1-J(A, B)=\frac{|A \cup B|-|A \cap B|}{|A \cup B|}$ 


\begin{tabular}{|c|c|c|c|c|c|c|c|c|c|c|c|c|c|c|c|c|c|c|c|c|c|c|c|}
\hline 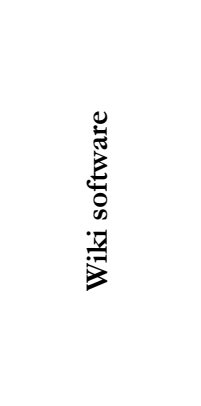 & 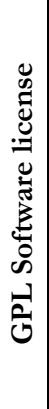 & 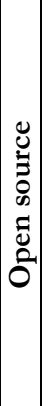 & 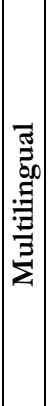 & 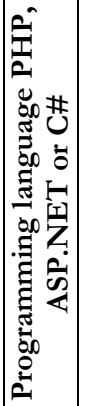 & 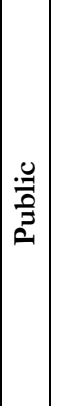 & 总 & 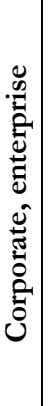 & 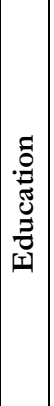 & $\begin{array}{l}\vec{\Xi} \\
\stackrel{\Xi}{\Xi} \\
\stackrel{\Xi}{\Xi}\end{array}$ & 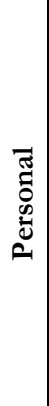 & 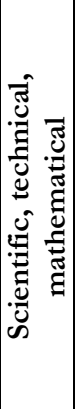 & 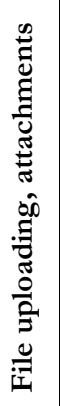 & 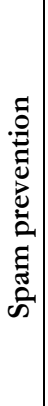 & 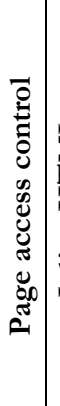 & 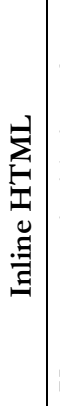 & 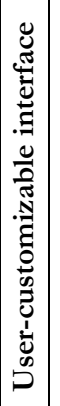 & 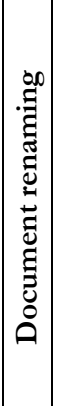 & 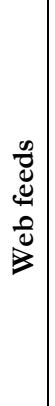 & 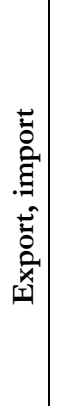 & 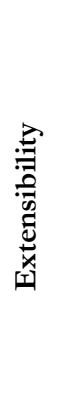 & 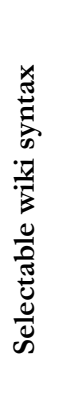 & 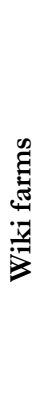 & 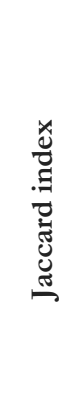 \\
\hline $\begin{array}{l}\text { BlueSpice } \\
\text { MediaWiki }\end{array}$ & 1 & 1 & 1 & 1 & 1 & 1 & 1 & 1 & 1 & & 1 & 1 & 1 & 1 & 1 & 1 & 1 & 1 & 1 & 1 & & 1 & 0.909 \\
\hline $\begin{array}{l}\text { Central } \\
\text { Desktop }\end{array}$ & & & & 1 & 1 & 1 & 1 & 1 & 1 & & & 1 & 1 & & 1 & 1 & & 1 & & & & & 0.500 \\
\hline $\begin{array}{l}\text { ConnectedTex } \\
\mathrm{t}\end{array}$ & & & 1 & & & 1 & & & & 1 & 1 & & & & 1 & 1 & 1 & 1 & 1 & 1 & & & 0.455 \\
\hline DokuWiki & 1 & 1 & 1 & 1 & 1 & 1 & 1 & 1 & 1 & 1 & 1 & 1 & 1 & 1 & 1 & 1 & 1 & 1 & 1 & 1 & 1 & 1 & 1.000 \\
\hline FlexWiki & & 1 & & 1 & 1 & 1 & 1 & & & & & 1 & 1 & 1 & 1 & 1 & 1 & 1 & & 1 & & & 0.591 \\
\hline Gitit & 1 & 1 & 1 & & 1 & 1 & 1 & 1 & 1 & 1 & 1 & 1 & 1 & 1 & 1 & 1 & 1 & 1 & 1 & 1 & 1 & 1 & 0.955 \\
\hline $\begin{array}{l}\text { IBM } \\
\text { Connections }\end{array}$ & & & 1 & & 1 & 1 & 1 & 1 & 1 & 1 & 1 & 1 & & & 1 & 1 & 1 & 1 & & 1 & 1 & 1 & 0.727 \\
\hline Midgard Wiki & 1 & 1 & & 1 & 1 & 1 & 1 & & & & & 1 & & 1 & 1 & 1 & & 1 & & 1 & & & 0.545 \\
\hline MindTouch & & & 1 & 1 & 1 & 1 & 1 & 1 & 1 & & & 1 & 1 & 1 & 1 & 1 & 1 & 1 & & 1 & & & 0.682 \\
\hline PBworks & & & & 1 & 1 & 1 & 1 & 1 & 1 & & & 1 & 1 & 1 & 1 & 1 & & 1 & & 1 & & & 0.591 \\
\hline PhpWiki & 1 & 1 & 1 & 1 & 1 & 1 & 1 & 1 & 1 & & & 1 & 1 & 1 & 1 & 1 & 1 & 1 & & 1 & 1 & & 0.818 \\
\hline PmWiki & 1 & 1 & 1 & 1 & 1 & 1 & 1 & 1 & 1 & 1 & 1 & 1 & 1 & 1 & 1 & 1 & 1 & 1 & 1 & 1 & 1 & 1 & 1.000 \\
\hline SharePoint & & & 1 & 1 & 1 & 1 & 1 & 1 & 1 & 1 & 1 & 1 & 1 & 1 & 1 & 1 & 1 & 1 & & 1 & 1 & 1 & 0.864 \\
\hline Socialtext & & & & & 1 & 1 & 1 & 1 & 1 & & & 1 & 1 & 1 & 1 & 1 & 1 & 1 & & 1 & & & 0.591 \\
\hline Swiki & 1 & 1 & & & 1 & 1 & & 1 & & & & 1 & 1 & 1 & 1 & 1 & 1 & & 1 & & & & 0.545 \\
\hline TiddlyWiki & & 1 & 1 & & & 1 & & & & 1 & & 1 & & & 1 & 1 & 1 & 1 & 1 & 1 & 1 & & 0.545 \\
\hline $\begin{array}{l}\text { Tiki Wiki } \\
\text { CMS } \\
\text { Groupware }\end{array}$ & 1 & 1 & 1 & 1 & 1 & 1 & 1 & 1 & 1 & 1 & 1 & 1 & 1 & 1 & 1 & 1 & 1 & 1 & & 1 & & 1 & 0.909 \\
\hline Trac & & 1 & 1 & & 1 & 1 & 1 & 1 & 1 & 1 & 1 & 1 & 1 & 1 & 1 & 1 & 1 & 1 & 1 & 1 & & & 0.818 \\
\hline TWiki & 1 & 1 & 1 & & 1 & 1 & 1 & 1 & 1 & 1 & 1 & 1 & 1 & 1 & 1 & 1 & 1 & 1 & & 1 & 1 & & 0.864 \\
\hline UseModWiki & 1 & 1 & & & 1 & 1 & 1 & 1 & 1 & & & 1 & 1 & 1 & 1 & 1 & & 1 & & 1 & & & 0.636 \\
\hline Wagn & 1 & 1 & 1 & & 1 & 1 & 1 & 1 & 1 & 1 & & 1 & 1 & 1 & 1 & 1 & 1 & 1 & & 1 & & & 0.773 \\
\hline Wiki.js & 1 & 1 & 1 & & 1 & 1 & 1 & 1 & 1 & 1 & 1 & 1 & 1 & 1 & 1 & 1 & 1 & & 1 & 1 & 1 & & 0.864 \\
\hline XWiki & 1 & 1 & 1 & & 1 & 1 & 1 & 1 & 1 & 1 & 1 & 1 & 1 & 1 & 1 & 1 & 1 & 1 & 1 & 1 & 1 & 1 & 0.955 \\
\hline ZWiki & 1 & 1 & 1 & & 1 & 1 & 1 & 1 & 1 & & & 1 & 1 & 1 & 1 & 1 & 1 & 1 & & 1 & & & 0.727 \\
\hline
\end{tabular}

\section{Discosure index computation (DIC) - Simple cardinal valuation method}

The decision maker establishes correlative relationships between the criteria ranked in importance, which progressively increase the share value associated. Evaluation algorithm is:

Step 1. Sort ascending criteria based on increased importance. Whether this order C1, C2, ..., Cn, (C 1 being the least important criterion).

Step 2. We attribute the weight criterion $\mathrm{C} 1$ value $\mathrm{x}, w_{1}:=x$.

Step 3. The decision maker considers the more important criterion $\mathrm{C} 2$ to $\mathrm{C} 1$ by objective determination of the value of the ratio

$$
\Delta w_{2}=\frac{w_{2}}{w_{1}}
$$

( $\Delta w_{2} \geq 1$ and equality occurs if one considers that $\mathrm{C} 1$ and $\mathrm{C} 2$ are of equal importance).

The process is applied to each criterion compared to the previous one. it

based on: $\Delta w_{j}$, for $j=2,3, \ldots, n$.

Step 4. The condition leads to the equation 


$$
\left(1+\Delta w_{2}+\Delta w_{2} \cdot \Delta w_{3}+\Delta w_{2} \cdot \Delta w_{3} \cdot \Delta w_{4}+\ldots+\Delta w_{2} \cdot \Delta w_{3} \cdot \ldots \cdot \Delta w_{n-1} \cdot \Delta w_{n}\right) \cdot x=1
$$

It must solve the equation and obtain the value of $\mathrm{x}$.

Step 5. It must calculate the weights of the n criteria:

$$
w_{j}=w_{j-1} \cdot \Delta w_{j}, \text { cu } j=2,3, \ldots, n \text { şi } w_{1}=x
$$

The importance of the criteria was established by the research team closely related to the characteristics of the problem to be solved, taking into account all factors of both internal and environmental nature that may influence the future operation of the platform.

\begin{tabular}{|c|c|c|}
\hline Criteria & $\begin{array}{c}\text { Criteria } \\
\text { importance }\end{array}$ & $\begin{array}{c}\text { The weights of } \\
\text { the criteria }\end{array}$ \\
\hline GPL Software license & 3 & 0.113 \\
\hline Open source & 3 & 0.113 \\
\hline Multilingual & 3 & 0.113 \\
\hline $\begin{array}{c}\text { Programming } \\
\text { language PHP, } \\
\text { ASP.NET or C\# }\end{array}$ & 3 & 0.113 \\
\hline Public & 3 & 0.113 \\
\hline Private & 2 & 0.038 \\
\hline Corporate & 2 & 0.038 \\
\hline Enterprise & 2 & 0.038 \\
\hline Education & 2 & 0.038 \\
\hline Intranet & 2 & 0.038 \\
\hline Personal & 2 & 0.038 \\
\hline $\begin{array}{c}\text { Scientific, technical, } \\
\text { mathematical }\end{array}$ & 2 & 0.038 \\
\hline File uploading & 1 & 0.019 \\
\hline Attachments & 1 & 0.019 \\
\hline Spam prevention & 1 & 0.019 \\
\hline Page access control & 1 & 0.019 \\
\hline Inline HTML & 1 & 0.019 \\
\hline User-customizable & 1 & 0.019 \\
\hline interface & 1 & 0.019 \\
\hline Document renaming & 1 & 0.019 \\
\hline Web feeds & 1 & 0.019 \\
\hline Export, import & 1 & 0.019 \\
\hline Extensibility & 1 & 0.019 \\
\hline Selectable wiki syntax & 1 & 0.019 \\
\hline Wiki farms & 1 & \\
\hline & & \\
\hline
\end{tabular}

If:

$$
\left(1+\Delta w_{2}+\Delta w_{2} \cdot \Delta w_{3}+\Delta w_{2} \cdot \Delta w_{3} \cdot \Delta w_{4}+\ldots+\Delta w_{2} \cdot \Delta w_{3} \cdot \ldots \cdot \Delta w_{24} \cdot \Delta w_{25}\right) \cdot x=1
$$

we solve the equation and obtain the value:

$$
\begin{aligned}
& \mathrm{x}=0.019 \\
& \text { Final Score }=\sum_{j=1}^{n} \text { Criteria Value } * w_{j}
\end{aligned}
$$




\begin{tabular}{|l|c|c|c|c|}
\hline \multicolumn{1}{|c|}{ Wiki software } & $\begin{array}{c}\text { Jaccard } \\
\text { Score }\end{array}$ & DIC Score & $\begin{array}{c}\text { Jaccard } \\
\text { Ranking }\end{array}$ & $\begin{array}{c}\text { DIC } \\
\text { Ranking }\end{array}$ \\
\hline DokuWiki & 1 & 1 & 1 & 1 \\
\hline PmWiki & 1 & 1 & 1 & 1 \\
\hline Tiki Wiki CMS Groupware & 0.909 & 0.962 & 2 & 2 \\
\hline BlueSpice MediaWiki & 0.909 & 0.943 & 2 & 3 \\
\hline Gitit & 0.955 & 0.887 & 3 & 4 \\
\hline PhpWiki & 0.818 & 0.887 & 4 & 4 \\
\hline XWiki & 0.955 & 0.887 & 5 & 4 \\
\hline TWiki & 0.864 & 0.849 & 6 & 5 \\
\hline Wiki.js & 0.864 & 0.849 & 6 & 5 \\
\hline Wagn & 0.773 & 0.792 & 7 & 6 \\
\hline SharePoint & 0.864 & 0.755 & 8 & 7 \\
\hline ZWiki & 0.727 & 0.755 & 9 & 7 \\
\hline Trac & 0.818 & 0.736 & 10 & 8 \\
\hline Midgard Wiki & 0.545 & 0.642 & 11 & 9 \\
\hline MindTouch & 0.682 & 0.642 & 12 & 9 \\
\hline UseModWiki & 0.636 & 0.623 & 13 & 10 \\
\hline IBM Connections & 0.727 & 0.604 & 14 & 11 \\
\hline FlexWiki & 0.591 & 0.566 & 15 & 12 \\
\hline Swiki & 0.545 & 0.547 & 16 & 13 \\
\hline PBworks & 0.591 & 0.509 & 17 & 14 \\
\hline Central Desktop & 0.500 & 0.472 & 18 & 15 \\
\hline TiddlyWiki & 0.545 & 0.453 & 19 & 16 \\
\hline Socialtext & 0.591 & 0.415 & 20 & 17 \\
\hline ConnectedText & 0.455 & 0.340 & 21 & 18 \\
\hline
\end{tabular}

Based on the Jaccard ranking and the DIC ranking, we note that for the identified requirements, based on their hierarchy, the most suitable platforms are DokuWiki and PmWiki.

\section{Conclusion}

For identifying the best platform, the authors propose a model based on both the Jaccard index and the Discosure index calculation (DIC) - Simple cardinal valuation method, depending on certain previously identified criteria.

Practically, starting from identifying requirements as well as their importance for a particular purpose, the model allows numerical comparison of multiple platforms.

Acknowledgement: This research was supported by The Project Future Web (PN-III-P1-1.2PCCDI-2017-0800). We thank our colleagues from Academy of Economic Studies and Dunarea de Jos University who provided insight and expertise that greatly assisted the research.

\section{References}

https://www.statisticshowto.datasciencecentral.com/jaccard-index/ https://www.sciencedirect.com/topics/computer-science/jaccard-coefficient https://neo4j.com/docs/graph-algorithms/current/algorithms/similarity-jaccard/ https://www.mathworks.com/help/images/ref/jaccard.html https://www.nap.edu/read/11186/chapter/6 